\title{
Upregulation of long non-coding RNA MALAT-1 confers poor prognosis and influences cell proliferation and apoptosis in acute monocytic leukemia
}

\author{
JIN-LONG HUANG, WEI LIU, LI-HONG TIAN, TING-TING CHAI, YANG LIU, FENG ZHANG, \\ HAI-YING FU, HUA-RONG ZHOU and JIAN-ZHEN SHEN \\ Fujian Institute of Hematology, Fujian Provincial Key Laboratory on Hematology, \\ Fujian Medical University Union Hospital, Fuzhou, Fujian 350001, P.R. China
}

Received March 1, 2017; Accepted June 9, 2017

DOI: $10.3892 /$ or.2017.5802

\begin{abstract}
Metastasis-associated lung adenocarcinoma transcript 1 (MALAT-1), a long non-coding RNA, has been documented to be a new prognostic marker and gene regulator in several types of cancer, but its potential involvement in acute myeloid leukemia (AML) remains unclear. This study investigated the expression and functional role of MALAT-1 in AML. MALAT-1 expression was assessed by real-time quantitative PCR. After lentiviral-mediated MALAT-1 knockdown, the proliferation of AML cells was determined by CCK- 8 and colony formation assays. Cell cycle progression and apoptosis were evaluated by flow cytometry and the expression of caspase- $3,-8$ and -9 was assessed by western blot analysis. We found that MALAT-1 expression in patients with acute monocytic leukemia (M5) was significantly increased when compared with that of healthy controls, and the overall survival of M5 patients with high MALAT-1 expression was markedly reduced when compared with the overall survival of patients with low MALAT-1 expression. The analysis
\end{abstract}

Correspondence to: Dr Jian-Zhen Shen, Fujian Institute of Hematology, Fujian Provincial Key Laboratory on Hematology, Fujian Medical University Union Hospital, 29 Xinquan Road, Fuzhou, Fujian 350001, P.R. China

E-mail: doctorjzs@163.com

Abbreviations: AL, acute leukemia; AML, acute myeloid leukemia; M0, acute myeloid leukemia with minimal differentiation; M1, AML with partial differentiation; M2, AML with maturation; M3, acute promyelocytic leukemia; M4, acute myelomonocytic leukemia with granules; M5, acute monocytic leukemia; M6, acute erythroleukemia; NC, negative control; ncRNA, non-coding RNA; lncRNA, long non-coding RNA; MALAT-1, metastasis-associated lung adenocarcinoma transcript 1; GAPDH, glyceraldehyde-3-phosphate dehydrogenase; RT-qPCR, real-time quantitative polymerase chain reaction

Key words: long non-coding RNA, metastasis-associated lung adenocarcinoma transcript 1 , acute myeloid leukemia, acute monocytic leukemia of cellular experiments showed that MALAT-1 silencing decreased the proliferation of M5 cells (U-937 and THP-1), inhibited cell cycle progression and increased apoptosis. Taken together, these findings suggest that high MALAT-1 expression is closely associated with poor prognosis in M5 patients and may play a role in leukemia cell proliferation and apoptosis, and may serve as a promising theranostic marker.

\section{Introduction}

Human genome sequence data have revealed that more than $90 \%$ of the human genome is transcribed mainly as non-coding RNAs (ncRNAs) and sparsely as protein-encoding RNAs ( $<2 \%$ ). Long non-coding RNAs (lncRNAs) are ncRNAs with transcripts greater than 200 nucleotides. They participate in different biological processes, including transcription, alternative splicing, epigenetic regulation, RNA decay, miRNA silencing, modulation of protein activity, structural and organizational roles of the cell, and alteration of protein localization (1). Recently, various lncRNAs have been proven to be closely related with both normal development and diseases, especially tumorigenesis (2). However, the mechanisms of action of most lncRNAs remain unclear $(1,3)$.

As a long intergenic non-coding RNA, metastasis-associated lung adenocarcinoma transcript 1 (MALAT-1) was identified in non-small cell lung cancer (NSCLC) (4), with a length of about $8,700 \mathrm{nt}$, and is located on human chromosome 11q13.1. MALAT-1 has been documented to be involved in a number of diseases, especially in the proliferation, invasion and metastasis of multiple types of cancers (5). Recent studies have reported its invasive and metastatic role in lung cancer $(4,6)$, hepatocellular carcinoma (7), gastric cancer (8), colorectal cancer (9) and bladder cancer (10). It has been generally accepted as an important marker to predict prognosis in earlystage lung adenocarcinoma or lung squamous carcinoma (4), pancreatic and renal cell carcinoma (11). It is also involved in cisplatin resistance in lung cancer (12). However, little literature is available to illustrate its role in acute leukemia.

Acute myeloid leukemia (AML) is a malignancy originating from the hematopoietic system and is characterized by clonal expansion of myeloid cells and a maturation arrest in bone 
marrow, which results in loss of normal hematopoietic function. As the most common type of acute leukemia in adults, the incidence of AML increases along with age and mainly affects middle-aged and elderly populations $(13,14)$. In recent years, complete remission (CR) and disease-free survival (DFS) of AML patients have been improved with the optimization of chemotherapy regimens and supportive treatments (15). However, $50-70 \%$ of AML patients who are able to achieve a CR with primary induction chemotherapy encounter relapse, with subsequent survival probability lowered to approximately $10 \%$ (16). Thus, most AML patients face a gloomy prognosis, and the identification of new predictive markers and therapeutic targets for AML is an urgent quest.

The present study aimed to investigate the potential role of MALAT-1 in AML. Firstly, we examined the expression levels of MALAT-1 in bone marrow samples of AML patients and healthy individuals. Second, we explored the possible mechanisms underlying of the affects of MALAT-1 on acute myeloid leukemia via cell experiments. The results showed that upregulation of MALAT-1 was closely associated with the poor prognosis of M5 patients and its knockdown prohibited the proliferation of M5 cell lines and promoted cell apoptosis, suggesting that MALAT-1 may serve as a potential prognostic marker and therapeutic target.

\section{Materials and methods}

Patients and clinical samples. Bone marrow cells were collected from 95 newly diagnosed AML patients (47 males and 48 females with a median age of 51 years; range, 14-89 years) admitted to the Affiliated Union Hospital of Fujian Medical University from December 2012 to November 2016. The diagnosis was classified according to the French-American-British (FAB) classification criteria and confirmed by morphology, immunophenotyping, cytogenetics, molecular cell biology (MICM classification criteria published by WHO) and additional examinations. Patients who underwent chemotherapy or radiotherapy prior to the study were excluded. Thirty-seven healthy bone marrow donors served as normal controls. Informed consent was obtained from the patients and healthy individuals before the use of these clinical samples and the study protocol was approved by the Ethics Committee of Affiliated Union Hospital. A telephone followup was conducted after obtaining consent from the patients' families, which was supplemented with the examination of inpatient and/or outpatient medical records.

After bone marrow puncture, 3-5 $\mathrm{ml}$ of the bone marrow was harvested. Mononuclear cells in bone marrow were extracted using lymphocyte separation medium (Hao Yang Bio, Tianjin, China). Cells were processed for extraction of DNA, RNA and proteins, or were stored at $-80^{\circ} \mathrm{C}$ for later use.

Cell culture. The acute monocytic leukemia cell lines, U-937 (catalog no. TCHu159) and THP-1 (catalog no. TCHu 57), were chosen according to the results of RT-qPCR. Both cell lines were obtained from the Cell Bank of the Chinese Academy of Sciences (Shanghai, China). All cell lines were maintained in RPMI-1640 medium (Hyclone, Logan, UT, USA) containing $10 \%$ fetal bovine serum (FBS; Hao Yang Bio) and maintained at $37^{\circ} \mathrm{C}$ in a humidified incubator containing $5 \% \mathrm{CO}_{2}$. The cells were passaged every two days, and those in logarithmic growth were used for further study.

Cell transfection. U-937 and THP-1 cell lines were used for further transfection experiments. Lentiviral vectors of MALAT-1 siRNA (si-MALAT-1) or scrambled negative control (si-NC) were designed and synthesized by Genechem (cat no. GIDL80771; Shanghai, China). Both U-937 and THP-1 cells were transfected with si-MALAT-1 and the si-NC lentivirus according to the manufacturer's instructions. The transfection efficiency was determined by fluorescence microscopy $96 \mathrm{~h}$ later.

Extraction of total cellular RNA and cDNA synthesis. Total RNA was extracted with TRIzol reagent (Invitrogen, Carlsbad, CA, USA). Two microliters of RNA were used to measure the concentration and purity on a quantitation analyzer (Thermo Fisher Scientific, Waltham, MA, USA). The purity of the RNA was within 1.8-2.0 and was confirmed by OD260/280 ratios. First-strand cDNA was synthesized using the RevertAid First-Strand cDNA Synthesis kit (Thermo Fisher Scientific). Reverse transcription was performed to generate complementary DNA in a final volume of $20 \mu \mathrm{l}$, containing $1 \mu \mathrm{g}$ of RNA, $1 \mu \mathrm{l}$ of random primer, $2 \mu \mathrm{l}$ of $\mathrm{dNTP} \operatorname{mix}(10 \mathrm{mM}), 1 \mu \mathrm{l}$ of RNase inhibiter, $1 \mu \mathrm{l}$ of reverse transcriptase, $4 \mu 1$ of $5 \mathrm{X}$ reverse transcriptase buffer and diethylpyrocarbonate (DEPC)-treated water. The procedure was performed according to the manufacturer's protocol (Thermo Fisher Scientific). The cDNA was either immediately used as a template for RT-qPCR or stored at $-20^{\circ} \mathrm{C}$ for later use.

Real-time quantitative reverse transcription polymerase chain reaction $(R T-q P C R)$ analysis of MALAT-1 expression. An ABI7500 real-time RCR module and FastStart Universal SYBR-Green Master kit (ROX; Roche, Indianapolis, IN, USA) were employed to investigate reverse-transcription products using glyceraldehyde-3-phosphate dehydrogenase (GAPDH) as the internal control. The primer sequences of MALAT-1 were as follows: forward, 5'-AAAGCAAGGTCTCCCCAC AAG-3' and reverse, 5'-GGTCTGTGCTAGATCAAAAG GCA-3'. The primer sequences of GAPDH were as follows: forward, 5'-CCCCTTCATTGACCTCAACTACAT-3' and reverse, 5'-CGCTCCTGGAAGATGGTGA-3'. The total reaction volume was $25 \mu \mathrm{l}$, including $12.5 \mu \mathrm{l}$ of SYBR-Green qPCR Mix (Roche), $1 \mu \mathrm{l}$ of cDNA, $0.75 \mu \mathrm{l}$ of forward and reverse primers at $10 \mu \mathrm{mol} / 1$, respectively, and $10 \mu \mathrm{l}$ of DEPC water. The reaction conditions were as follows: $50^{\circ} \mathrm{C}$ for $2 \mathrm{~min}$ for 1 cycle (first stage); $95^{\circ} \mathrm{C}$ for $2 \mathrm{~min}$ for 1 cycle (second stage); followed by $95^{\circ} \mathrm{C}$ for $15 \mathrm{sec}$ and then $60^{\circ} \mathrm{C}$ for $30 \mathrm{sec}$ for 40 cycles (third stage). Melting curve analysis involved one cycle of $95^{\circ} \mathrm{C}$ for $15 \mathrm{sec} ; 60^{\circ} \mathrm{C}$ for $1 \mathrm{~min} ; 95^{\circ} \mathrm{C}$ for $15 \mathrm{sec}$; and then $60^{\circ} \mathrm{C}$ for $15 \mathrm{sec}$. Every reaction mixture was triplicated and RT-qPCR was also tripled for each sample and all cell groups. The expression levels of MALAT-1 were normalized to the internal control GAPDH reference to obtain the relative threshold cycle $(\Delta \mathrm{Ct})$. The relative levels were calculated by the comparative $\mathrm{Ct}(\Delta \Delta \mathrm{Ct})$ method, and the relative expression fold $\left(2^{-\Delta \Delta C t}\right)$ was calculated.

Cell proliferation assay and colony formation analysis. Cell proliferation rates were measured using Cell Counting Kit- 8 
(CCK-8; Dojindo, Japan) according to the manufacturer's instructions. U-937 cells were seeded into 96-well plates at a density of $7 \times 10^{3}$ cells $/ 100 \mu 1$ and THP-1 cells at a density of $2.5 \times 10^{4}$ cells $/ 100 \mu \mathrm{l}$. In each plate, there were three groups (3 wells/group): blank control group (no cells but medium), negative control group (U-937/THP-1 cells transfected with si-NC), and the experimental group (U-937/THP-1 cells transfected with si-MALAT-1). Cell proliferation was determined after 24, 48, 72 and $96 \mathrm{~h}$, respectively. An amount of $10 \mu \mathrm{l}$ of CCK-8 solution was added to each well. Cells were incubated at $37^{\circ} \mathrm{C}$ for $2 \mathrm{~h}$, and then the optical density (OD) was measured with a microplate reader (Biotek, Richmond, VT, USA) at a wavelength of $450 \mathrm{~nm}$ using the following formula: $\mathrm{OD}_{\text {experiment }}-\mathrm{OD}_{\text {blank }}$. The experiment was repeated three times.

To assess the colony formation, U-937 and THP-1 cells transfected with si-NC or si-MALAT-1 lentivirus were seeded into 24-well plates (500 $\mu \mathrm{l} /$ well, 200 cells/well, three wells/group), which were pre-coated with methyl cellulose solution (500 $\mu \mathrm{l} /$ well). The cells were then incubated at $37^{\circ} \mathrm{C}$ in a humidified incubator containing 5\% $\mathrm{CO}_{2}$. After 10-14 days, the colonies were counted under an inverted microscope (Nikon, Tokyo, Japan). A colony was defined as an aggregate of $>40$ cells. The methyl cellulose solution was prepared for use as mentioned in our previous study (17).

Analysis of the cell cycle and apoptosis. Cell cycle and apoptosis analyses were performed on a BD FACSVerse ${ }^{\mathrm{TM}}$ flow cytometer (BD Biosciences, San Jose, CA, USA) according to the manufacturer's instructions.

Analysis of the cell cycle. Cells $\left(1 \times 10^{6}\right)$ were collected after a 96-h transfection and washed with cold phosphate-buffered saline (PBS), and then fixed in $70 \%$ ethanol at $4^{\circ} \mathrm{C}$ overnight. After fixation, the cells were washed again and resuspended in PBS, and then stained with PI/RNase Staining Buffer (BD Biosciences) in the dark at $25^{\circ} \mathrm{C}$ for $15 \mathrm{~min}$, and finally analyzed with a flow cytometer (BD Biosciences) and Modlfit software (Verity Software House, Topsham, ME, USA).

Analysis of apoptosis. After 96-h transfection, cell apoptosis was analyzed with the PE Annexin V Apoptosis Detection kit I (BD Biosciences) according to the manufacturer's protocol. Cells were discriminated into viable cells, dead cells, early apoptotic and late apoptotic cells. Ratios of early and late apoptotic cells were compared with those of the control group. Results were analyzed with FlowJo software (BD Biosciences). All experiments were performed in triplicate.

In addition, cells were collected and treated with bisbenzimide (Hoechst 33258; Beyotime, Shanghai, China), following the manufacturer's instructions. Apoptotic cells were observed under a fluorescence microscope (Nikon). Each experiment was conducted in triplicate.

Western blot analysis. Protein was extracted using lysis solution containing protease inhibitors (all from Lulong Biotech, Fujian, China). Equal amounts of total protein $(80 \mu \mathrm{g})$ from every sample were separated by $10 \%$ SDS-PAGE (Beyotime). The proteins were then transferred onto PVDF membranes (0.2 $\mu \mathrm{m}$; Millipore, Bedford, MA, USA), which were blocked with skim milk (Boster, Hubei, China), and then incubated with the following primary antibodies at $4{ }^{\circ} \mathrm{C}$ overnight: GAPDH (Abcam, Cambridge, MA, USA), caspase-3, caspase- 8 and caspase-9 (Cell Signaling Technology, Danvers, MA, USA). After being washed with TBST, the membranes were incubated with secondary antibodies (Cell Signaling Technology) at room temperature for $2 \mathrm{~h}$ and the signals of the membranes were detected with West Pico Chemiluminescent substrate (Thermo Fisher Scientific). Band intensities were analyzed with ImageJ software (1.45s; National Institutes of Health Bethesda, MD, USA). The expression levels of proteins were normalized to the internal control GAPDH. The experiment was repeated three times.

Statistical analysis. Data are expressed as mean \pm SD and were analyzed with SPSS19.0 Statistical Software (IBM, Armonk, NY, USA) and GraphPad Prism 6.0 (GraphPad Software, Inc., La Jolla, CA, USA). Results of IncRNA MALAT-1 expression in clinical samples were assessed by nonparametric Mann-Whitney U-test, and multiple groups were compared using Kruskal-Wallis $\mathrm{H}$ test. Three independent experiments were performed for all measurements. The significance of mean differences between two groups in the cellular function experiments was calculated by unpaired two-tailed Student's t-tests. Rates were compared and analyzed by the Chi-square or Fisher's exact tests. The overall survival (OS) was estimated by the Kaplan-Meier method and log-rank test. Variables influencing OS significantly in univariate analysis were analyzed by using a Cox proportional hazards model. OS was measured from the beginning of the study to the death of the patient (by any cause) or to the last follow-up time for surviving patients. $\mathrm{P}<0.05$ was deemed statistically significant.

\section{Results}

Expression of MALAT-1 in bone marrow cells derived from AML patients, and analysis of clinical features and prognosis. The RT-qPCR analysis showed that the expression of MALAT-1 in M5 patients was markedly increased when compared with that of the healthy controls $(\mathrm{p}<0.01)$ (Fig. 1A) and non-M5 AML patients $(\mathrm{p}<0.01)$ (Fig. 1B), while no significant difference was observed when other AML subtypes (M0, M1, M2, M3, M4 or M6) were compared with the controls ( $p>0.05$, respectively) (Fig. 1A). No significant difference was observed between the non-M5 AML patients and the controls $(\mathrm{p}=0.5398)$ (Fig. 1B). The clinicopathological characteristics of all AML patients are summarized in Table $\mathrm{I}$.

The markedly increased expression of MALAT-1 in M5 patients necessitated a probe into its clinical relevance in these patients. Fifty-six M5 patients (27 males and 29 females with a median age of 51 years; range, 14-89 years) were included for further analysis. The correlation between MALAT-1 expression and clinicopathological parameters was then assessed (Table II). The M5 patients were divided into two groups using the median MALAT-1 $2^{-\Delta \mathrm{Ct}}$ value of 17.4118 (Fig. 2A). Those with an MALAT-1 expression level lower than the median value (17.4118) were assigned to the low-expression group $(n=28)$, and those with expression above the median value was assigned to the high-expression group $(n=28)$. As shown in Table II, a high MALAT-1 level was associated with higher white blood cell count $(\mathrm{p}<0.01)$ and 

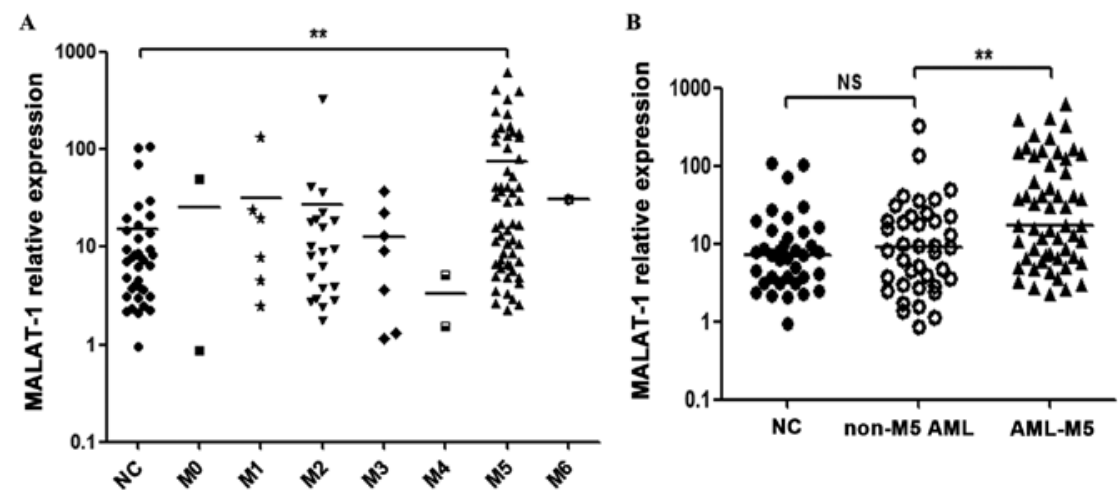

Figure 1. Expression levels of MALAT-1 in AML patients and healthy controls by RT-qPCR analysis (2-4Ct) (normalized to GAPDH). (A) Increased expression of MALAT-1 in M5 patients when compared with controls (** $\mathrm{p}<0.01$; Kruskal-Wallis $\mathrm{H}$ test). (B) Increased expression of MALAT-1 in M5 patients when compared with non-M5 AML patients (" $\mathrm{p}<0.01$; Mann-Whitney U test). No obvious difference in MALAT-1 expression between non-M5 AML patients and controls was observed ( $\mathrm{p}=0.5398$; Mann-Whitney U test). MALAT-1, metastasis-associated lung adenocarcinoma transcript 1; AML, acute myeloid leukemia; GAPDH, glyceraldehyde-3-phosphate dehydrogenase; NS, not significant.

Table I. Clinicopathological characteristics of the AML patients.

AML subtypes

\begin{tabular}{|c|c|c|c|c|c|c|c|}
\hline \multirow{2}{*}{ Factors } & \multicolumn{7}{|c|}{ AML subtypes } \\
\hline & $\begin{array}{c}\text { M0 } \\
\mathrm{n}\end{array}$ & $\begin{array}{c}\text { M1 } \\
n\end{array}$ & $\begin{array}{c}\text { M2 } \\
n\end{array}$ & $\begin{array}{c}\text { M3 } \\
n\end{array}$ & $\begin{array}{c}\text { M4 } \\
\mathrm{n}\end{array}$ & M5 & $\begin{array}{c}\text { M6 } \\
\text { n }\end{array}$ \\
\hline \multicolumn{8}{|l|}{ Sex } \\
\hline Male & 1 & 1 & 15 & 1 & 1 & 27 & 0 \\
\hline Female & 1 & 5 & 6 & 6 & 1 & 29 & 1 \\
\hline \multicolumn{8}{|l|}{ Age (years) } \\
\hline$<60$ & 2 & 3 & 15 & 3 & 2 & 37 & 1 \\
\hline$\geq 60$ & 0 & 3 & 6 & 4 & 0 & 19 & 0 \\
\hline \multicolumn{8}{|l|}{$\mathrm{WBC}\left(\mathrm{x} 10^{9} / 1\right)$} \\
\hline$<100$ & 2 & 3 & 16 & 6 & 2 & 40 & 1 \\
\hline$\geq 100$ & 0 & 3 & 5 & 1 & 0 & 16 & 0 \\
\hline \multicolumn{8}{|l|}{ HB (g/l) } \\
\hline$<60$ & 0 & 1 & 3 & 1 & 2 & 10 & 0 \\
\hline$\geq 60$ & 2 & 5 & 18 & 6 & 0 & 46 & 1 \\
\hline \multicolumn{8}{|l|}{$\operatorname{PLT}\left(x 10^{9} / 1\right)$} \\
\hline$<30$ & 0 & 2 & 9 & 5 & 2 & 19 & 0 \\
\hline$\geq 30$ & 2 & 4 & 12 & 2 & 0 & 37 & 1 \\
\hline \multicolumn{8}{|l|}{$\begin{array}{l}\text { Bone marrow } \\
\text { blasts (\%) }\end{array}$} \\
\hline$<60$ & 1 & 0 & 9 & 0 & 1 & 13 & 1 \\
\hline$\geq 60$ & 1 & 6 & 12 & 7 & 1 & 43 & 0 \\
\hline \multicolumn{8}{|c|}{$\begin{array}{l}\text { Risk stratification } \\
\text { based on karyotype }\end{array}$} \\
\hline Better & 0 & 0 & 2 & 6 & 0 & 2 & 0 \\
\hline Intermediate & 2 & 4 & 14 & 0 & 2 & 20 & 0 \\
\hline Poor & 0 & 0 & 0 & 0 & 0 & 4 & 0 \\
\hline Unkown & 0 & 2 & 5 & 1 & 0 & 30 & 1 \\
\hline
\end{tabular}

${ }^{\text {a} B e t t e r, ~ i n v(16), ~ o r ~} \mathrm{t}(16 ; 16) ; \mathrm{t}(8 ; 21) ; \mathrm{t}(15 ; 17)$. Intermediate, normal cytogenetics; +8 alone; $\mathrm{t}(9 ; 11)$; other non-defined. Poor, complex $(\geq 3$ clonal chromosomal abnormalities); monosomal karyotype, -5, 5q-, -7, 7q-; 11q23-non t(9;11); inv(3), t(3;3); t(6;9); t(9;22). AML, acute myeloid leukemia; WBC, white blood cell count; HB, hemoglobin; PLT, platelets.
Table II. MALAT-1 expression and clinicopathological characteristics of the M5 patients.

\begin{tabular}{|c|c|c|c|}
\hline Factors & $\begin{array}{c}\text { MALAT-1 } \\
\text { high expression } \\
(n=28) n\end{array}$ & $\begin{array}{c}\text { MALAT-1 } \\
\text { low expression } \\
(n=28) n\end{array}$ & $\mathrm{P}$-value \\
\hline \multicolumn{4}{|l|}{ Sex } \\
\hline Male & 13 & 14 & \multirow[t]{2}{*}{0.789} \\
\hline Female & 15 & 14 & \\
\hline \multicolumn{4}{|l|}{ Age (years) } \\
\hline$<60$ & 20 & 17 & \multirow[t]{2}{*}{0.397} \\
\hline$\geq 60$ & 8 & 11 & \\
\hline \multicolumn{4}{|l|}{ WBC $\left(\times 10^{9} / 1\right)$} \\
\hline$<100$ & 15 & 25 & \multirow[t]{2}{*}{0.003} \\
\hline$\geq 100$ & 13 & 3 & \\
\hline \multicolumn{4}{|l|}{$\mathrm{HB}(\mathrm{g} / \mathrm{l})$} \\
\hline$<60$ & 6 & 4 & \multirow[t]{2}{*}{0.485} \\
\hline$\geq 60$ & 22 & 24 & \\
\hline \multicolumn{4}{|l|}{ PLT (x10 $\left.10^{9}\right)$} \\
\hline$<30$ & 5 & 14 & \multirow[t]{2}{*}{0.011} \\
\hline$\geq 30$ & 23 & 14 & \\
\hline \multicolumn{4}{|l|}{$\begin{array}{l}\text { Bone marrow } \\
\text { blasts }(\%)\end{array}$} \\
\hline$<60$ & 4 & 9 & \multirow[t]{2}{*}{0.114} \\
\hline$\geq 60$ & 24 & 19 & \\
\hline \multicolumn{4}{|c|}{$\begin{array}{l}\text { Risk stratification } \\
\text { based on karyotype }\end{array}$} \\
\hline Better & 1 & 1 & \multirow[t]{4}{*}{0.424} \\
\hline Intermediate & 12 & 8 & \\
\hline Poor & 3 & 1 & \\
\hline Unkown & 12 & 18 & \\
\hline
\end{tabular}

${ }^{a}$ Better, inv(16), or $\mathrm{t}(16 ; 16) ; \mathrm{t}(8 ; 21) ; \mathrm{t}(15 ; 17)$. Intermediate, normal cytogenetics; +8 alone; $\mathrm{t}(9 ; 11)$; other non-defined. Poor, complex $(\geq 3$ clonal chromosomal abnormalities); monosomal karyotype, -5, 5q-, -7, 7q-; 11q23-non t(9;11); inv(3), t(3;3); t(6;9); t(9;22). MALAT-1, metastasis-associated lung adenocarcinoma transcript 1 ; WBC, white blood cell; HB, hemoglobin; PLT, platelets. 
A

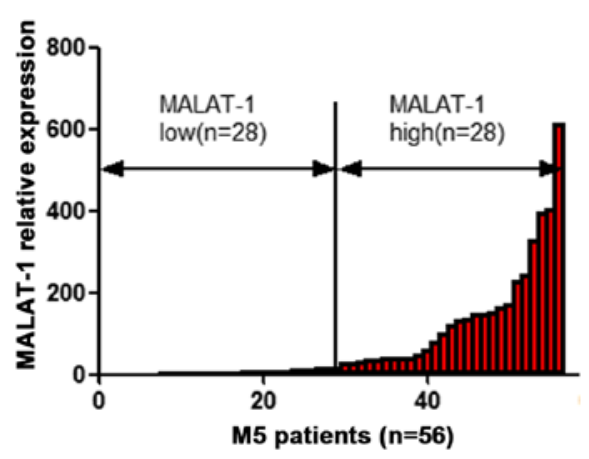

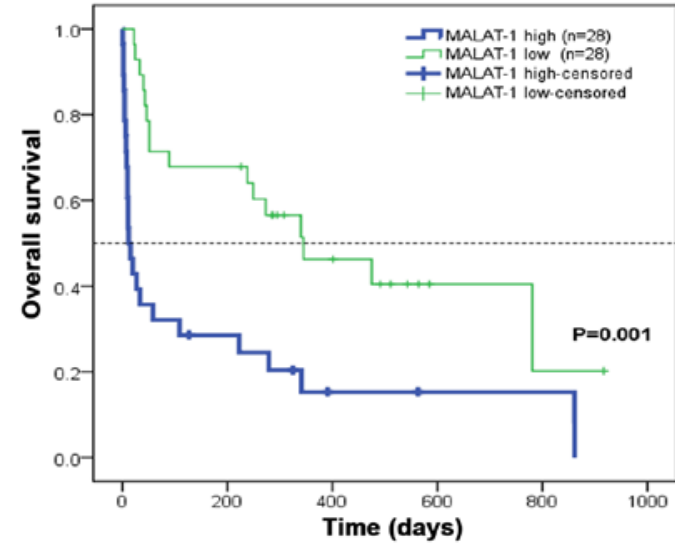

Figure 2. OS of M5 patients. (A) M5 patients were divided into two groups based on MALAT-1 expression levels; high-expression MALAT-1 group (n=28) and low-expression MALAT-1 group (n=28). (B) Kaplan-Meier method was used to analyze the association between expression level of MALAT-1 and OS in M5 patients. The OS of the high-expression group was significantly lower than that of the low-expression group ( $\mathrm{p}=0.001, \log$-rank test). MALAT-1, metastasisassociated lung adenocarcinoma transcript 1 ; OS, overall survival.

Table III. Univariate analysis of OS of the patients with AML-M5 (n=56).

\begin{tabular}{lccc}
\hline Variables & Group & $\mathrm{n}$ & P-value (OS) \\
\hline Sex & Male/female & $27 / 29$ & 0.526 \\
Age $($ years $)$ & $<60 / \geq 60$ & $37 / 19$ & 0.218 \\
WBC $\left(\times 10^{9} / 1\right)$ & $<100 / \geq 100$ & $40 / 16$ & 0.006 \\
HB $(\mathrm{g} / \mathrm{l})$ & $<60 / \geq 60$ & $10 / 46$ & 0.866 \\
PLT $\left(\times 10^{9} / 1\right)$ & $<30 / \geq 30$ & $19 / 37$ & 0.754 \\
Bone marrow blasts & $<60 \% / \geq 60 \%$ & $13 / 43$ & 0.921 \\
MALAT-1 & High/low & $28 / 28$ & 0.001 \\
\hline
\end{tabular}

AML, acute myeloid leukemia; WBC, white blood cell count; HB, hemoglobin; PLT, platelets; MALAT-1, metastasis-associated lung adenocarcinoma transcript 1; OS, overall survival.

higher platelet count $(\mathrm{p}<0.05)$. However, it was not directly associated with other clinical parameters, including sex, age, hemoglobin level, bone marrow blast count and stratification based on karyotype.

The follow-up was completed on November 22, 2016. The Kaplan-Meier survival curve was used to analyze the association of MALAT-1 expression with the OS of M5 patients. The results revealed that, compared with the patients with low MALAT-1 expression, OS of the high-expression group was significantly decreased $(\mathrm{p}<0.01)$ (Fig. $2 \mathrm{~B})$. The mean OS over 5 years (2012-2016) was estimated at $182.24 \pm 60.558$ days [95\% confidence interval (CI), 63.547-300.933] for the high-expression group and at $449.285 \pm 72.462$ days $(95 \% \mathrm{CI}$, 307.259-591.311) for the low-expression group. Clinical prognostic indicators were also analyzed. Univariate analysis of OS showed that white blood cell count and MALAT-1 expression level were prognostic indicators while the remaining clinical parameters were not (Table III). In the multivariate analysis, the Cox proportional hazards model revealed that MALAT-1 overexpression was an independent prognostic
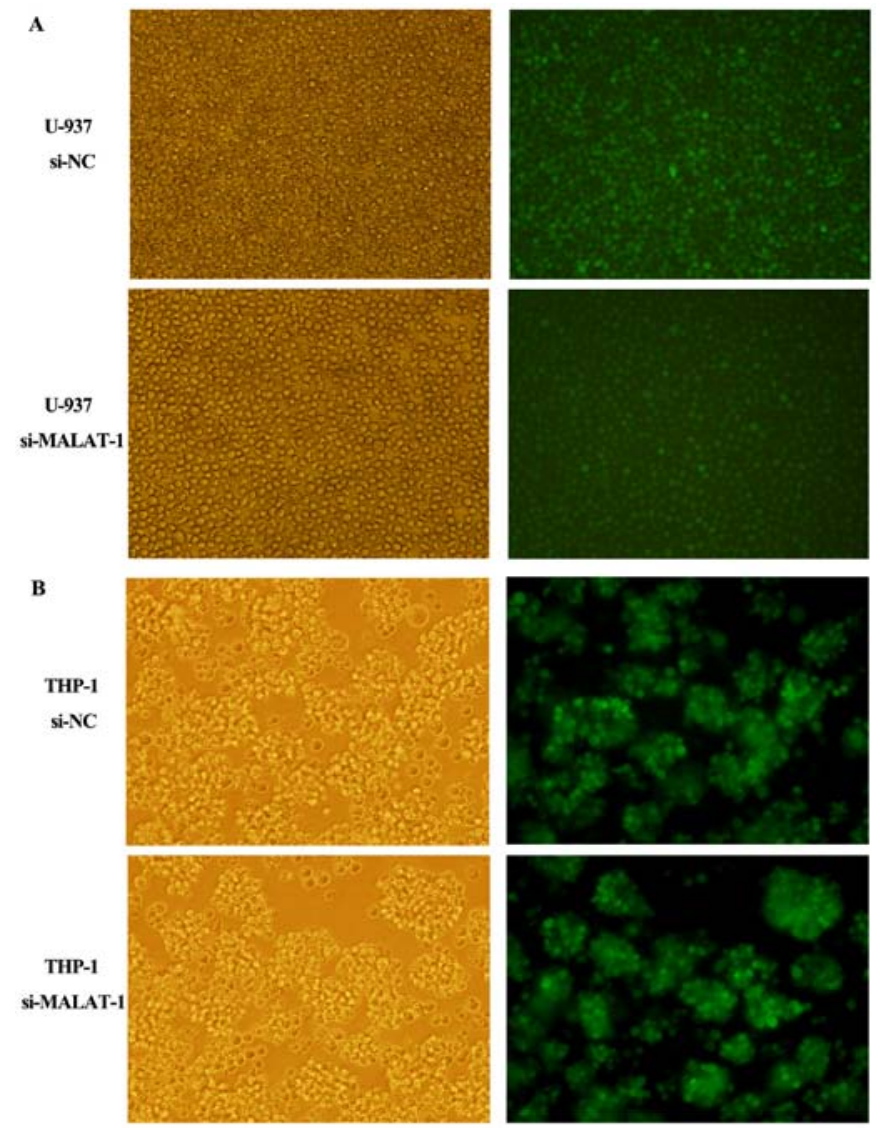

Figure 3. Transfection efficiency of U-937 and THP-1 cells. Both U-937 and THP-1 cells were transfected with si-MALAT-1 and si-NC lentivirus. The transfection efficiency was determined by fluorescence microscopy $96 \mathrm{~h}$ later as shown in A (U-937) and B (THP-1). MALAT-1, metastasis-associated lung adenocarcinoma transcript 1 .

factor for OS [hazard ratio (HR), 2.551; 95\% CI, 1.309-4.97; $\mathrm{p}=0.006]$ (Table IV).

Effects of MALAT-1 knockdown on proliferation, colony formation and cell cycle of U-937 and THP-1 cells. Due to 
A
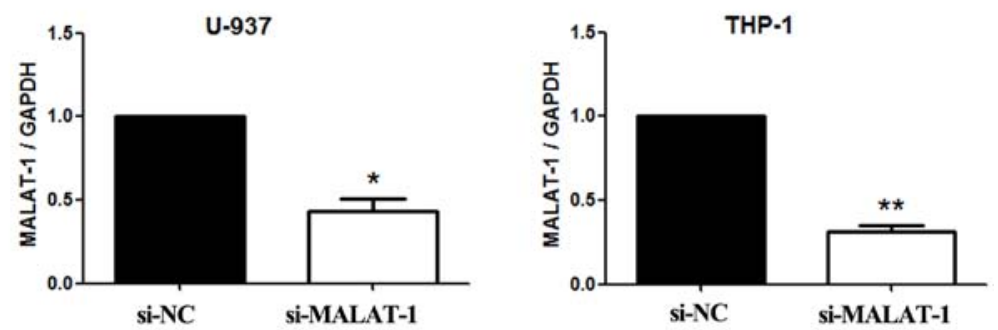

B
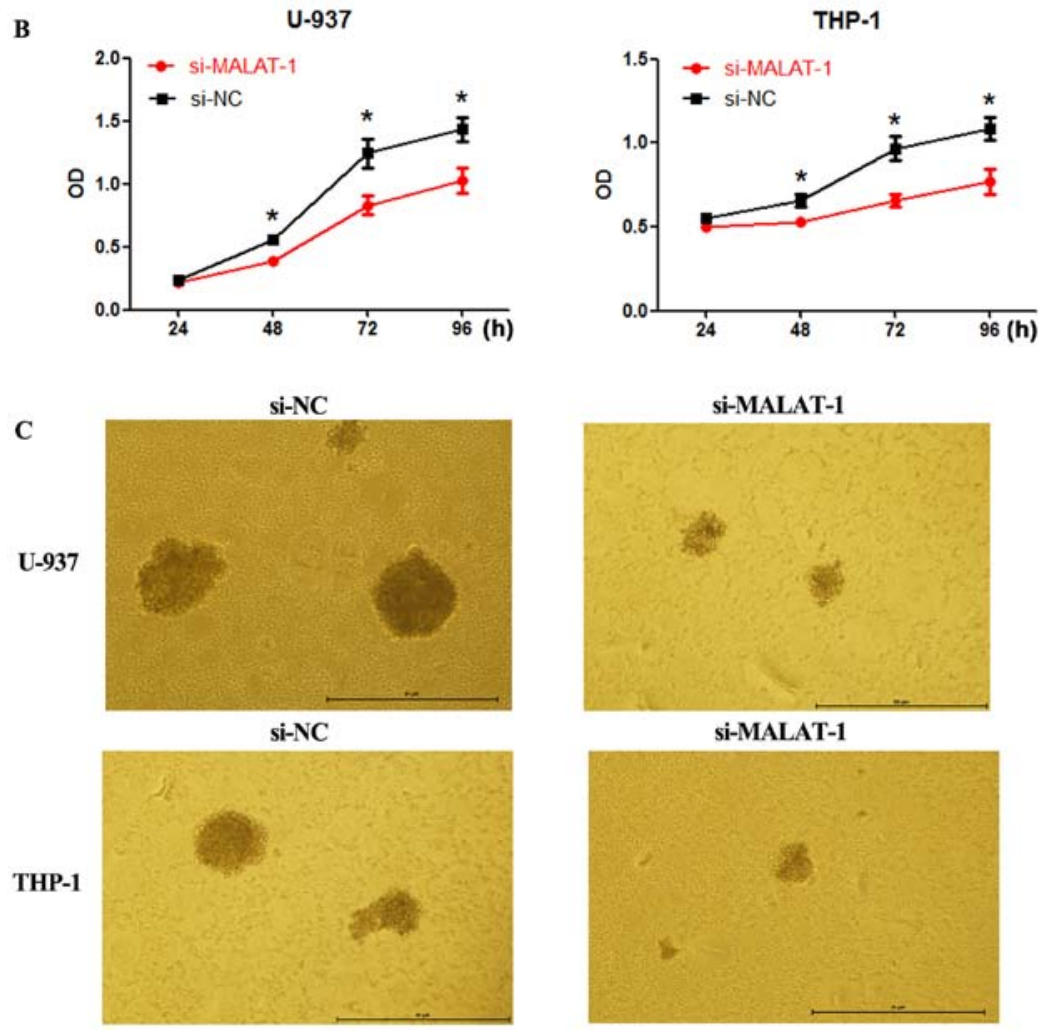

Figure 4. Effects of MALAT-1 knockdown on M5 cell function (U-937, THP-1). (A) RT-qPCR analysis was used to assess the expression levels of MALAT-1 in U-937 and THP-1 cells after transfection ( ${ }^{*} \mathrm{p}<0.05 ;{ }^{* *} \mathrm{p}<0.01$; unpaired two-tailed Student's t-tests). Effects of MALAT-1 knockdown on (B) cell proliferation and $(\mathrm{C})$ colony formation in si-NC cells and si-MALAT-1 cells ( $\mathrm{p} p<0.05$; unpaired two-tailed Student's t-tests). MALAT-1, metastasis-associated lung adenocarcinoma transcript 1.

its abnormal expression in M5 clinical samples, we speculated that MALAT-1 may play a role in M5 progression. To assess the role of MALAT-1 in M5 cellular growth, we silenced MALAT-1 expression in the U-937 and THP-1 cell lines with small interfering RNA (siRNA). The transfection efficiency was determined by fluorescence microscopy $96 \mathrm{~h}$ later as shown in Fig. 3A (U-937) and B (THP-1). The siRNA which decreased MALAT-1 expression level by more than $50 \%$ was chosen for further experiments (Fig. 4A). Cells transfected with si-NC were regarded as the control.

As presented in the results of the CCK8 assays (Fig. 4B), cell growth was suppressed in both cell lines transfected with si-MALAT-1 when compared with cell growth of the si-NC groups. In addition, colony formation in the U-937 and THP-1 cells was also reduced by MALAT-1 silencing (Fig. 4C). Cell cycle progression in the U-937 and THP-1 cells was further analyzed by flow cytometry. In both cell lines, compared with the si-NC groups, a significantly greater proportion of G0/G1 phase cells was observed among the MALAT-1 knockdown groups (U-937, p<0.01; THP-1, p<0.05) (Fig. 5). The si-NC
Table IV. Multivariate analysis of OS of patients with AML-M5 $(n=56)$.

\begin{tabular}{lcccccc}
\hline & & & \multicolumn{4}{c}{ OS } \\
\cline { 5 - 7 } Variables & Group & $\mathrm{n}$ & HR & $95 \%$ CI & P-value \\
\hline WBC $\left(\times 10^{9} / 1\right)$ & $<100 / \geq 100$ & $40 / 16$ & 1.911 & $0.934-3.910$ & 0.076 \\
MALAT-1 & High/low & $28 / 28$ & 2.551 & $1.309-4.970$ & 0.006 \\
\hline
\end{tabular}

OS, overall survival; AML, acute myeloid leukemia; HR, hazard ratio; CI, confidence interval; WBC, white blood cell count; MALAT-1, metastasis-associated lung adenocarcinoma transcript 1.

group had an increased proportion of cells in the $\mathrm{S}$ phase in both cell lines (U-937, p<0.001; THP-1, p<0.01) (Fig. 5).

Effects of MALAT-1 knockdown on U-937 and THP-1 cell apoptosis. We next investigated whether MALAT-1 knockdown 

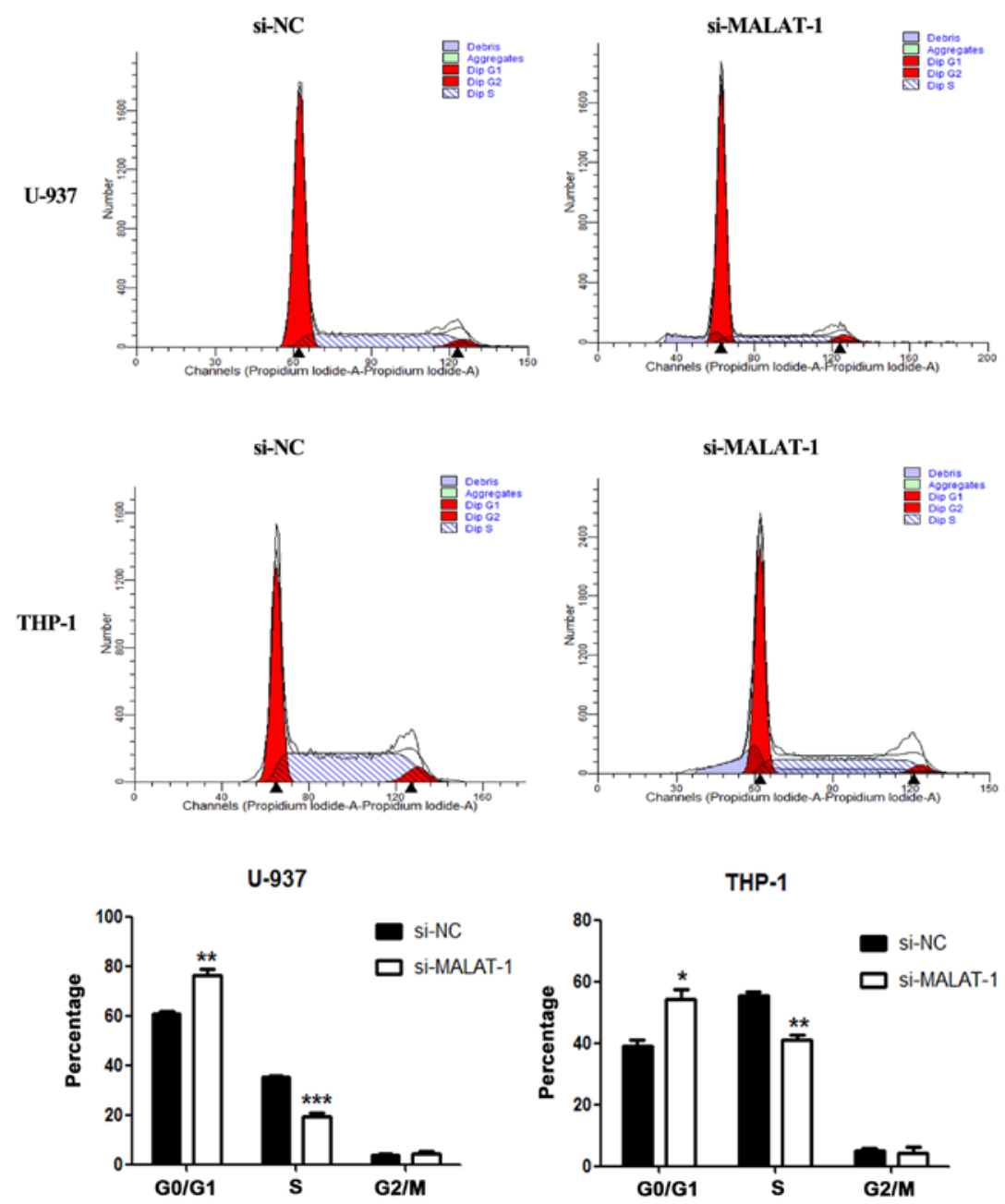

Figure 5. Effects of MALAT-1 knockdown on cell cycle distribution in si-NC and si-MALAT-1 cells $\left({ }^{*} \mathrm{p}<0.05 ;{ }^{* *} \mathrm{p}<0.01 ;{ }^{* * *} \mathrm{p}<0.001 ;\right.$ unpaired two-tailed Student's t-tests). MALAT-1, metastasis-associated lung adenocarcinoma transcript 1.

affects cell apoptosis. Flow cytometric analysis revealed that the percentage of apoptotic U-937 cells was significantly increased in the MALAT-1-silenced group when compared with that of the si-NC group ( $\mathrm{p}<0.001)$ (Fig. 6). A similar result was observed in the THP-1 cells $(\mathrm{p}<0.01)$ (Fig. 6).

In addition, Hoechst 33258 staining was applied to confirm cell apoptosis. In the U-937 and THP-1 cell lines, few apoptotic cells were observed in the si-NC group (Fig. 7A, left panel). However, in the MALAT-1-silenced cells, the nuclei were dense and stained, and some nuclei were disintegrated and fragmented into dense granular particles (apoptotic bodies) (Fig. 7A, arrows, right panel).

Finally, the effects of the silencing of MALAT-1 on the activation of the apoptosis-associated proteins, caspase-3, caspase- 8 , caspase- 9 , were also determined by western blotting. Western blot analysis showed that compared with those of the si-NC groups, protein expression levels of caspase-3, caspase- 8 and caspase- 9 were upregulated in the MALAT-1silenced U-937 and THP-1 cells (Fig. 7B).

\section{Discussion}

The present study focused on the roles of lncRNA MALAT-1 in acute myeloid leukemia. A significantly upregulated expression of MALAT-1 was found in the M5 patient tissues when compared with the healthy controls and non-M5 AML patient tissues, while no statistical difference was found in tissues of other AML subtypes. Moreover, MALAT-1 silencing inhibited the proliferation and cycle progression of M5 cells, which also increased the apoptosis. Compared with other AML subtypes, M5 patients frequently report a high incidence of hyperleukocytosis, extramedullary infiltration, disorders of hemostasis, low complete remission (CR) rate for treatment and poor prognosis $(18,19)$. Thus, the identification of MALAT-1 in M5 may contribute to promising strategies for the diagnosis and therapy of AML.

MALAT-1 is broadly expressed in human tissues including lymphatic tissues, bone marrow and lymphocytes $(20,21)$. In a recent study of newly diagnosed patients with multiple myeloma, the expression of MALAT-1 was found to be strongly correlated with disease status and thus may serve as a predictor of early progression, suggesting its role in the pathogenesis of myeloma (22). Another recent study reported a strong association between expression levels of lncRNAs (e.g., MALAT-1, TUG1, HOTAIR, lincRNA-p21) and the clinical stages of chronic lymphocytic leukemia (CLL) (23). In addition, MALAT-1 may play a role in vincristine resistance in childhood acute lymphoblastic leukemia (24). These 

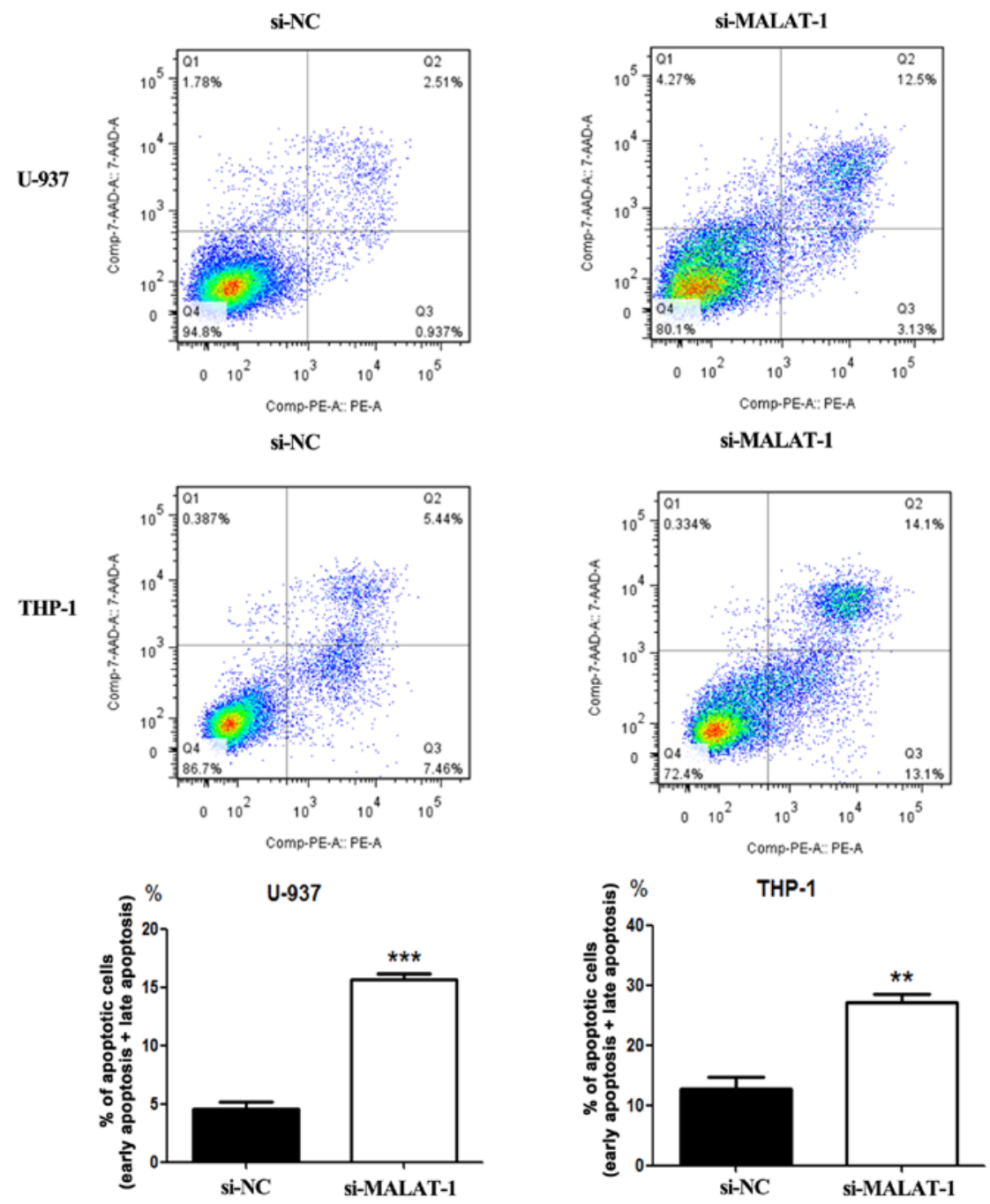

Figure 6. Effects of MALAT-1 knockdown on cell apoptosis. Apoptosis of the si-NC and si-MALAT-1 groups was evaluated by flow cytometry after Annexin V and PI staining $\left({ }^{* *} \mathrm{p}<0.01 ;{ }^{* * *} \mathrm{p}<0.001\right.$; unpaired two-tailed Student's t-tests). MALAT-1, metastasis-associated lung adenocarcinoma transcript 1.

findings on hematopoietic system cancers lend support to the present study and hint at the necessity of exploring the role of MALAT-1 in acute leukemia.

In present study, MALAT-1 expression was upregulated in M5 patients and was significantly associated with higher white blood cell count, which is consistent with other research on solid tumors and myeloma (11) and in agreement with a recent study regarding the roles of IncRNA HOTAIR in acute leukemia (12). However, statistical analysis also revealed a correlation between upregulated MALAT-1 and a higher platelet level, which was not in accordance with the previous related evidence on HOTAIR $(25,26)$. We speculate that this inconsistency may result from the insufficient number of cases or that MALAT-1 may have a special role in the hematopoietic system. Previous literature has documented that MALAT-1 expression is an independent prognostic parameter for survival in several types of cancers (5). Similarly, univariate and multivariate analyses in the present research demonstrated that high MALAT-1 expression, instead of other clinical characteristics, was highly correlated with poor OS. These findings indicate that MALAT-1 may serve as a candidate indicator for evaluating the prognosis of M5 patients. It should not be ignored that statistical difference was not found in other AML subtypes when compared with the control, which may be due to the limited number of cases. Both a larger cohort and a longer follow-up period would be needed in further research.

We also speculate that MALAT-1 may function as an oncogene in the development of M5. To confirm the hypothesis, we analyzed the effects of MALAT-1 knockdown on the proliferation and apoptosis of U-937 and THP-1 cell lines. We found that the downregulation of MALAT-1 in M5 cells inhibited the cell proliferation, colony formation and cell cycle progression by inducing G0/G1 stage arrest, which is consistent with some previous studies $(27,28)$. Silencing of MALAT-1 in gastric cancer cell line SGC-7901 was found to inhibit cancer progression by inducing G0/G1 arrest (27), and a similar result was observed in cervical cancer cell line OVCAR3 (28). MALAT-1 knockdown was found to induce upregulated protein expression of cyclin E and cyclin-dependent kinase 2 (CDK2) by silencing tumor suppressor gene p53 and activating transcription factor $\mathrm{E} 2 \mathrm{~F}$, as a consequence of $\mathrm{G} 1 / \mathrm{S}$ phase transition to $\mathrm{G} 2$ phase (29). Moreover, a reduction in mRNA levels of B-MYB and CENPE was observed in MALAT1-depleted cells, thus influencing mitotic progression and cell cycle (29). However, it is still unclear whether MALAT-1 influences cell proliferation by interacting with several cyclins or cyclin-dependent kinases 
A
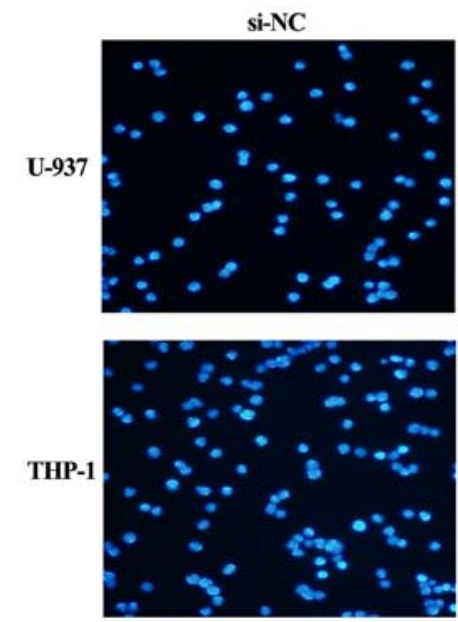

B
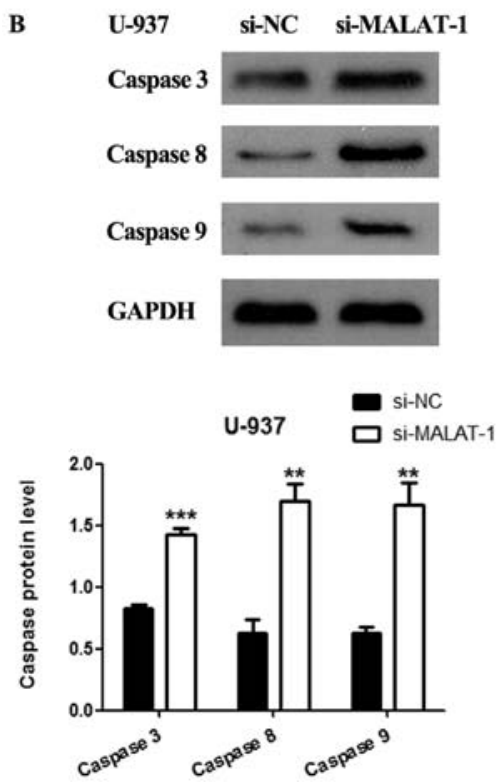
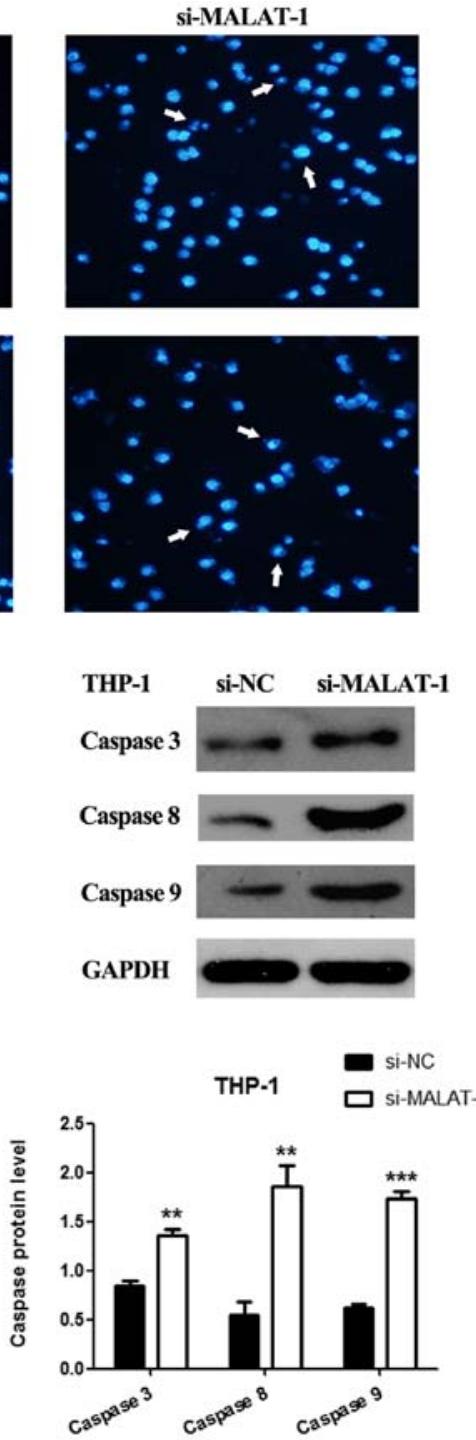

Figure 7. Hoechst 33258 staining and western blot analysis of cells. (A) Apoptosis of the si-NC and si-MALAT-1 groups was evaluated using Hoechst 33258 staining, and several apoptotic cells are indicated with white arrows. (B) Western blot analysis of the protein levels (caspase-3, caspase-8 and caspase-9) in the si-NC and si-MALAT-1 groups ${ }^{* * *} \mathrm{p}<0.01 ;{ }^{* * * *} \mathrm{p}<0.001$; unpaired two-tailed Student's t-tests). MALAT-1, metastasis-associated lung adenocarcinoma transcript 1 .

(CDKs) in leukemia, and further studies are awaited to elucidate the specific molecular mechanisms.

In addition to reduced cell proliferation, MALAT-1 knockdown increased the apoptosis ratio in both U-937 and THP-1 cells, which was confirmed by Hoechst 33258 staining and flow cytometric analysis. Furthermore, western blot analysis revealed that protein expression of caspase-3, caspase- 8 and caspase- 9 was upregulated in the MALAT-1-knockdown cells. Apoptosis-associated proteins play important roles in apoptotic signaling pathways, which include initiator caspases (caspase-8, and caspase-9) and executioner caspases (caspase-3, and caspase-7). Caspase-3, caspase- 8 and caspase- 9 are key members in the regulation of programmed cell death (30). Our finding is consistent with a study of cervical cancer by Guo et al (31), in which MALAT-1 knockdown upregulated the mRNA expression of pro-apoptotic genes, caspase-3, caspase- 8 and Bax, and suppressed expression of anti-apoptotic genes, $\mathrm{Bcl}-2$ and $\mathrm{Bcl}-\mathrm{xl}$. Our results suggest that knockdown of MALAT-1 may induce apoptosis by activating caspase-related apoptotic signaling pathways in leukemia. Further studies are needed to investigate whether MALAT-1 plays a role in regulating the expression of upstream genes ( $\mathrm{Bcl}-2$ or $\mathrm{Bax}$ ) in the caspase pathway, and the underlying mechanism.

In summary, the present study demonstrated that a high expression of lncRNA MALAT-1 in acute monocytic leukemia is related to clinicopathologic parameters including patient outcome. Experiments in vitro showed that knockdown of MALAT-1 inhibited leukemia cell proliferation and induced apoptosis, thus indicating that MALAT-1 may serve as a promising biomarker of poor prognosis and a therapeutic target for M5 treatment. However, several limitations are present in the study: a small number of cases were assessed in the research; the mechanisms by which MALAT-1 was upregulated in M5 patients were not assessed; and no in vivo experiments were conducted to throw light upon the molecular mechanism involved in acute leukemia progression. Further studies are warranted to clarify the role of MALAT-1 in various subtypes of leukemia and its therapeutic potential and prognostic value in leukemia. 


\section{Acknowledgements}

The present study was sponsored by the National Natural Science Foundation of China (no. 81370629), Fujian Provincial Environmental Protection and Technology Program (2015R011), and the National and Fujian Provincial Key Clinical Specialty Discipline Construction Program (China).

\section{References}

1. Sana J, Faltejskova P, Svoboda M and Slaby O: Novel classes of non-coding RNAs and cancer. J Transl Med 10: 103, 2012.

2. Ponting CP, Oliver PL and Reik W: Evolution and functions of long noncoding RNAs. Cell 136: 629-641, 2009.

3. Qiu MT, Hu JW, Yin R and Xu L: Long noncoding RNA: An emerging paradigm of cancer research. Tumour Biol 34: 613-620, 2013.

4. Ji P, Diederichs S, Wang W, Böing S, Metzger R, Schneider PM, Tidow N, Brandt B, Buerger H, Bulk E, et al: MALAT-1, a novel noncoding RNA, and thymosin beta4 predict metastasis and survival in early-stage non-small cell lung cancer. Oncogene 22 8031-8041, 2003.

5. Shi X, Sun M, Liu H, Yao Y and Song Y: Long non-coding RNAs: A new frontier in the study of human diseases. Cancer Lett 339: 159-166, 2013.

6. Shen L, Chen L, Wang Y, Jiang X, Xia H and Zhuang Z: Long noncoding RNA MALAT1 promotes brain metastasis by inducing epithelial-mesenchymal transition in lung cancer. J Neurooncol 121: 101-108, 2015.

7. Lai MC, Yang Z, Zhou L, Zhu QQ, Xie HY, Zhang F, Wu LM, Chen LM and Zheng SS: Long non-coding RNA MALAT-1 overexpression predicts tumor recurrence of hepatocellular carcinoma after liver transplantation. Med Oncol 29: 1810-1816, 2012.

8. Okugawa Y, Toiyama Y, Hur K, Toden S, Saigusa S, Tanaka K, Inoue Y, Mohri Y, Kusunoki M, Boland CR, et al: Metastasis-associated long non-coding RNA drives gastric cancer development and promotes peritoneal metastasis. Carcinogenesis 35: 2731-2739, 2014.

9. Ji Q, Zhang L, Liu X, Zhou L, Wang W, Han Z, Sui H, Tang Y, Wang Y, Liu N, et al: Long non-coding RNA MALAT1 promotes tumour growth and metastasis in colorectal cancer through binding to SFPQ and releasing oncogene PTBP2 from SFPQ/PTBP2 complex. Br J Cancer 111: 736-748, 2014.

10. Han Y, Liu Y, Nie L, Gui Y and Cai Z: Inducing cell proliferation inhibition, apoptosis, and motility reduction by silencing long noncoding ribonucleic acid metastasis-associated lung adenocarcinoma transcript 1 in urothelial carcinoma of the bladder. Urology 81: 209.e1-209.e7, 2013.

11. Tian X and $\mathrm{Xu}$ G: Clinical value of lncRNA MALAT1 as a prognostic marker in human cancer: Systematic review and meta-analysis. BMJ Open 5: e008653, 2015.

12. Lopez-Ayllon BD, Moncho-Amor V, Abarrategi A, Ibañez de Cáceres I, Castro-Carpeño J, Belda-Iniesta C, Perona R and Sastre L: Cancer stem cells and cisplatin-resistant cells isolated from non-small-lung cancer cell lines constitute related cell populations. Cancer Med 3: 1099-1111, 2014.

13. Dores GM, Devesa SS, Curtis RE, Linet MS and Morton LM: Acute leukemia incidence and patient survival among children and adults in the United States, 2001-2007. Blood 119: 34-43, 2012.

14. Löwenberg B, Downing JR and Burnett A: Acute myeloid leukemia. N Engl J Med 341: 1051-1062, 1999.
15. Farag SS, Ruppert AS, Mrózek K, Mayer RJ, Stone RM, Carroll AJ, Powell BL, Moore JO, Pettenati MJ, Koduru PR, et al: Outcome of induction and postremission therapy in younger adults with acute myeloid leukemia with normal karyotype: A cancer and leukemia group B study. J Clin Oncol 23: 482-493, 2005.

16. Mims A and Stuart RK: Developmental therapeutics in acute myelogenous leukemia: Are there any new effective cytotoxic chemotherapeutic agents out there? Curr Hematol Malig Rep 8: 156-162, 2013.

17. Shen JZ,Zhang YY,Fu HY, Wu DS and Zhou HR: Overexpression of microRNA-143 inhibits growth and induces apoptosis in human leukemia cells. Oncol Rep 31: 2035-2042, 2014.

18. Azoulay E, Fieux F, Moreau D, Thiery G, Rousselot P, Parrot A, Le Gall JR, Dombret H and Schlemmer B: Acute monocytic leukemia presenting as acute respiratory failure. Am J Respir Crit Care Med 167: 1329-1333, 2003.

19. Porcu P, Cripe LD, Ng EW, Bhatia S, Danielson CM, Orazi A and McCarthy LJ: Hyperleukocytic leukemias and leukostasis: A review of pathophysiology, clinical presentation and management. Leuk Lymphoma 39: 1-18, 2000.

20. Lane L, Argoud-Puy G, Britan A, Cusin I, Duek PD, Evalet O, Gateau A, Gaudet P, Gleizes A, Masselot A, et al: neXtProt: A knowledge platform for human proteins. Nucleic Acids Res 40 (D1): D76-D83, 2012.

21. Wu C, Macleod I and Su AI: BioGPS and MyGene.info: Organizing online, gene-centric information. Nucleic Acids Res 41 (D1): D561-D565, 2013.

22. Cho SF, Chang YC, Chang CS, Lin SF, Liu YC, Hsiao HH, Chang JG and Liu TC: MALAT1 long non-coding RNA is overexpressed in multiple myeloma and may serve as a marker to predict disease progression. BMC Cancer 14: 809, 2014.

23. Isin M, Ozgur E, Cetin G, Erten N, Aktan M, Gezer U and Dalay $\mathrm{N}$ : Investigation of circulating $\operatorname{lncRNAs}$ in B-cell neoplasms. Clin Chim Acta 431: 255-259, 2014.

24. Akbari Moqadam F, Lange-Turenhout EA, Ariës IM, Pieters R and den Boer ML: miR-125b, miR-100 and miR-99a co-regulate vincristine resistance in childhood acute lymphoblastic leukemia. Leuk Res 37: 1315-1321, 2013.

25. Wu S, Zheng C, Chen S, Cai X, Shi Y, Lin B and Chen Y: Overexpression of long non-coding RNA HOTAIR predicts a poor prognosis in patients with acute myeloid leukemia. Oncol Lett 10: 2410-2414, 2015.

26. Zhang YY, Huang SH, Zhou HR, Chen CJ, Tian LH and Shen JZ: Role of HOTAIR in the diagnosis and prognosis of acute leukemia. Oncol Rep 36: 3113-3122, 2016.

27. Wang J, Su L, Chen X, Li P, Cai Q, Yu B, Liu B, Wu W and Zhu Z: MALAT1 promotes cell proliferation in gastric cancer by recruiting SF2/ASF. Biomed Pharmacother 68: 557-564, 2014.

28. Zhou Y, Xu X, Lv H, Wen Q, Li J, Tan L, Li J and Sheng X: The long noncoding RNA MALAT-1 is highly expressed in ovarian cancer and induces cell growth and migration. PLoS One 11: e0155250, 2016.

29. Tripathi V, Shen Z, Chakraborty A, Giri S, Freier SM, Wu X, Zhang Y, Gorospe M, Prasanth SG, Lal A, et al: Long noncoding RNA MALAT1 controls cell cycle progression by regulating the expression of oncogenic transcription factor B-MYB. PLoS Genet 9: e1003368, 2013.

30. Miura M: Apoptotic and nonapoptotic caspase functions in animal development. Cold Spring Harb Perspect Biol 4: 4, 2012.

31. Guo F, Li Y, Liu Y, Wang J, Li Y and Li G: Inhibition of metastasisassociated lung adenocarcinoma transcript 1 in CaSki human cervical cancer cells suppresses cell proliferation and invasion. Acta Biochim Biophys Sin (Shanghai) 42: 224-229, 2010. 